\title{
Intestinal Stricture
}

National Cancer Institute

\section{Source}

National Cancer Institute. Intestinal Stricture. NCI Thesaurus. Code 698962.

Fibrosis of the wall of a segment of the intestine that leads to intestinal lumen narrowing. 